\title{
Deep Learning for Image-Based Cassava Disease Detection
}

\author{
Amanda Ramcharan ${ }^{1}$, Kelsee Baranowski ${ }^{1}$, Peter McCloskey ${ }^{2}$, Babuali Ahmed ${ }^{3}$, \\ James Legg ${ }^{3}$ and David P. Hughes ${ }^{1,4,5 *}$ \\ ${ }^{1}$ Department of Entomology, College of Agricultural Sciences, Penn State University, State College, PA, United States, \\ ${ }^{2}$ Department of Computer Science, Pittsburgh University, Pittsburgh, PA, United States, ${ }^{3}$ International Institute of Tropical \\ Agriculture, Dar el Salaam, Tanzania, ${ }^{4}$ Department of Biology, Eberly College of Sciences, Penn State University, State \\ College, PA, United States, ${ }^{5}$ Center for Infectious Disease Dynamics, Huck Institutes of Life Sciences, Penn State University, \\ State College, PA, United States
}

Cassava is the third largest source of carbohydrates for human food in the world but is vulnerable to virus diseases, which threaten to destabilize food security in sub-Saharan Africa. Novel methods of cassava disease detection are needed to support improved control which will prevent this crisis. Image recognition offers both a cost effective and scalable technology for disease detection. New deep learning models offer an avenue for this technology to be easily deployed on mobile devices. Using a dataset of cassava disease images taken in the field in Tanzania, we applied transfer learning to train a deep convolutional neural network to identify three diseases and two types of pest damage (or lack thereof). The best trained model accuracies were $98 \%$ for brown leaf spot (BLS), 96\% for red mite damage (RMD), 95\% for green mite damage (GMD), 98\% for cassava brown streak disease (CBSD), and 96\% for cassava mosaic disease (CMD). The best model achieved an overall accuracy of $93 \%$ for data not used in the training process. Our results show that the transfer learning approach for image recognition of field images offers a fast, affordable, and easily deployable strategy for digital plant disease detection.

Keywords: cassava disease detection, deep learning, convolutional neural networks, transfer learning, mobile epidemiology, Inception v3 model

\section{INTRODUCTION}

Cassava (Manihot esculenta Crantz) is the most widely grown root crop in the world and a major source of calories for roughly two out of every five Africans (Nweke et al., 2002). In 2014, over 145 million tonnes of cassava were harvested on 17 million hectares of land on the African continent (FAOSTAT, 2017). It is considered a food security crop for smallholder farms, especially in lowincome, food-deficit areas (Bellotti et al., 1999) as it provides sufficient yields in low soil fertility conditions and where there are irregular rainfall patterns (De Bruijn and Fresco, 1989).

Smallholder farmers, representing $85 \%$ of the world's farms, face numerous risks to their agricultural production such as climate change, market shocks, and pest and disease outbreaks (Nagayet, 2005). Cassava, an exotic species introduced to Africa from South America in the 16th century, initially had few pest and disease constraints on the continent. In the 1970s two arthropod pests, the cassava mealybug [Phenacoccus manihoti(Matt.-Ferr.)] and the cassava green mite [Mononychellus tanajoa (Bond.)] were accidentally introduced from the neotropics (Legg, 1999), becoming the most economically threatening pests. Cassava virus diseases, in particular cassava mosaic disease (CMD) and cassava brown streak disease (CBSD), have a longer history on 
the continent. Mosaic disease was the first to be recorded in Tanzania towards the end of the 19th century (Warburg, 1894). In East Africa, the outbreak of a severe form of the virus in the 1990s, termed East African cassava mosaic virus (EACMV-UG or $\mathrm{UgV}$ ), coupled with the sensitivity of local cultivars, resulted in a threat to food security in the region as farmers' only solution was to abandon cultivation (Thresh et al., 1994). Thresh et al. (1997) estimated annual yield losses to CMD at $15-24 \%$, or $21.8-34.8$ million tons, at 1994 production levels. CBSD was reported in the 1930s (Storey, 1936). With limited success in controlling CMD and CBSD, the two diseases have become the largest constraints to cassava production and food security in sub-Saharan Africa resulting in losses of over US\$1 billion every year (Legg et al., 2006).

In order to manage the detection and spread of cassava diseases, early identification in the field is a crucial first step. Traditional disease identification approaches rely on the support of agricultural extension organizations, but these approaches are limited in countries with low logistical and human infrastructure capacity, and are expensive to scale up. In such areas, internet penetration, smartphone and unmanned aerial vehicle (UAV) technologies offer new tools for in-field plant disease detection based on automated image recognition that can aid in early detection at a large scale. Previous research has demonstrated automated image recognition of crop diseases in wheat (Gibson et al., 2015; Siricharoen et al., 2016), apples (Dubey and Jalal, 2014) and on datasets of healthy and diseased plants (Mohanty et al., 2016); this technology was also demonstrated on UAVs (Puig et al., 2015). Cassava disease detection based on automated image recognition through feature extraction has shown promising results (Aduwo et al., 2010; Abdullakasim et al., 2011; Mwebaze and Owomugisha, 2016) but extracting features is computationally intensive and requires expert knowledge for robust performance. In order to capitalize on smartphone technology, models must be fast and adapted to limited processing power. Transfer learning, where a model that has been trained on a large image dataset is retrained for new classes, offers a shortcut to training deep learning models because of lower computational requirements. This would have a distinct advantage for field settings. Here we investigated the potential for adapting an already trained deep learning convolutional neural network model to detect incidence of cassava disease using an infield dataset of 2,756 images comprising 3 cassava diseases and 2 types of pest damage (or lack thereof).

\section{METHODS}

\subsection{The Cassava Image Datasets}

The cassava leaf images were taken with a commonly available Sony Cybershot 20.2-megapixel digital camera in experimental fields belonging to the International Institute of Tropical Agriculture (IITA), outside of Bagamoyo, Tanzania. The entire cassava leaf roughly centered in the frame was photographed to build the first dataset (Figure S6). Over a four-week period, 11,670 images were taken. Images of cassava diseases were taken using several cassava genotypes and stages of maturity (as described in Table S1) in order to provide the full range of symptoms for each given disease to the deep learning model. Each of the diseases or types of pest damage was distinctive and the variation of symptom expression between varieties was minor in comparison to the contrasts between diseases.

Images were then screened for co-infections to limit the number of images with multiple diseases. This dataset, called the "original cassava dataset," comprised 2,756 images. These photos were then manually cropped into individual leaflets to build the second dataset. This dataset, called the "leaflet cassava dataset," comprised 15,000 images of cassava leaflets (2,500 images per class). Figure 1 shows an example from both datasets: (a) original cassava dataset and (b) leaflet cassava dataset and Figures S1S5 show examples from both datasets for each cassava disease. Both datasets were tested to shed light on model performance with images of full leaves but fewer images versus cropped leaves with more images. The underlying assumption was that the cropped leaf images (leaflet cassava dataset) would improve model performance to correctly identify a disease as the dataset was larger. Additionally, we suspected the end users trying to get a diagnosis for a disease would focus in on leaflets showing symptoms. Both datasets comprised six class labels assigned manually based on in-field diagnoses by cassava disease experts from IITA. For all datasets, we used the images as is, in color, and with varied backgrounds from the field in order to assess the model performance (Figure S6).

The six class labels for the datasets included three disease classes, two mite damage classes, and one healthy class, defined as a lack of disease or mite damage on the leaf. The disease classes and the number of images in the original dataset were: cassava brown streak disease (CBSD) (398 images), cassava mosaic disease (CMD) (388 images), brown leaf spot (BLS) (386 images), and the mite damage classes were: cassava green mite damage (GMD) (309 images) and red mite damage (RMD) (415 images). Figure 2 illustrates examples of the class labels for the original cassava dataset. Within these classes, several cassava varieties were photographed at different stages of plant maturity (Table S1).

The five disease and pest class symptoms are as follows:

CBSD is a result of infection with cassava brown streak ipomoviruses (CBSIs) (family Potyviridae, genus Ipomovirus). There are two species associated with the disease, Cassava brown streak virus (CBSV) and Ugandan cassava brown streak virus (UCBSV) (Mbanzibwa et al., 2011). Both cause the same symptoms. These two virus species are vectored by whiteflies [Bemisia tabaci (Genn.)] in a semi-persistent manner. When infected, cassava leaves show a mottled yellowing pattern typically beginning from the secondary veins and progressing to tertiary veins as the infection gets more severe (Nichols, 1950). This yellowish chlorosis spreads along the veins until severely infected leaves are mostly yellow. Disease symptoms can vary by variety, age of the plant and weather conditions. Tolerant varieties and plants at a young age may be infected but asymptomatic. The two viruses may also cause brown streaks on stems of infected plants and brown necrotic rotting in tuberous roots which may render them inedible.

CMD is a result of infection with cassava mosaic begomoviruses (CMBs) (family Geminiviridae, genus 

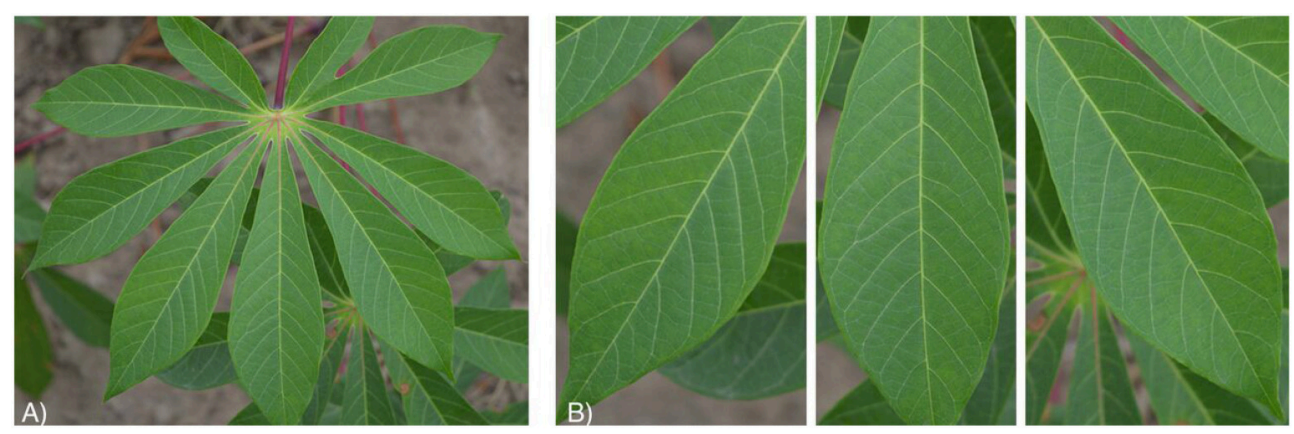

FIGURE 1 | Healthy cassava leaf images from the (A) original cassava dataset and (B) leaflet dataset.
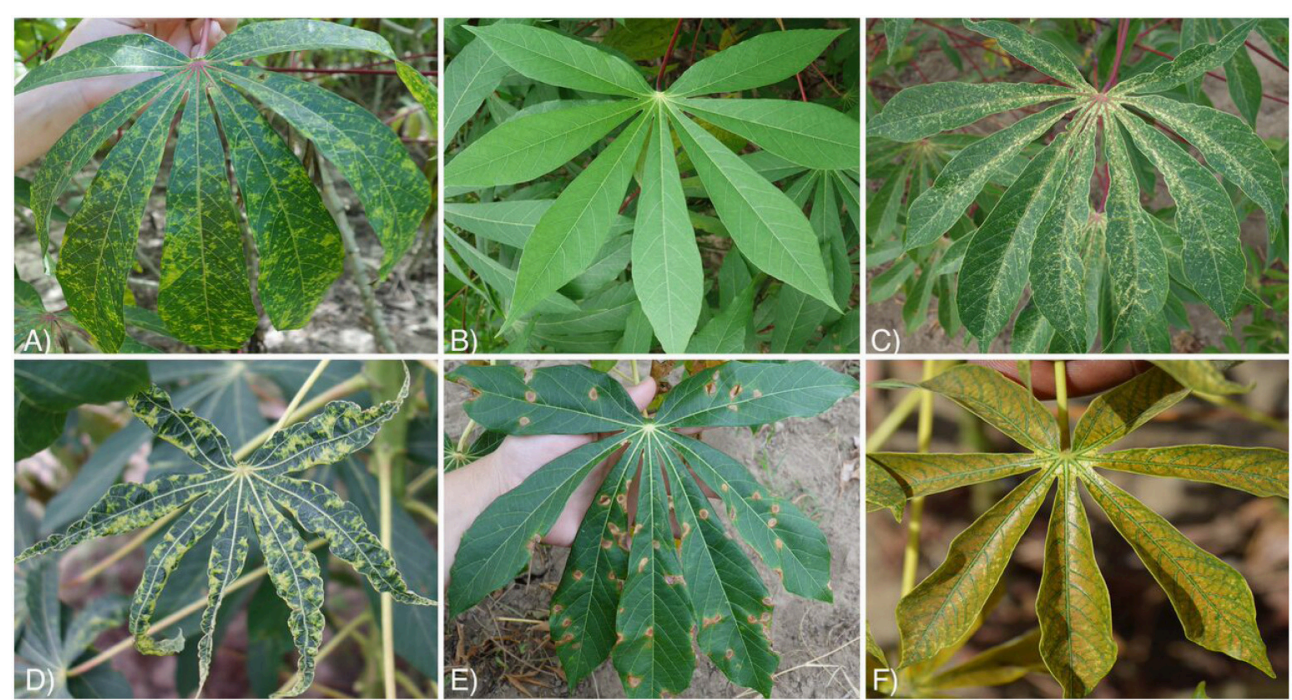

FIGURE 2 | Examples of images with in field backgrounds from 6 classes in the original cassava dataset. (A) Cassava brown streak disease (CBSD), (B) Healthy, (C) Green mite damage (GMD), (D) Cassava mosaic disease (CMD), (E) Brown leaf spot (BLS), (F) Red mite damage (RMD).

Begomovirus). There are many species and recombinant strains associated with this group of viruses, although the common form in coastal East Africa, where sampling was undertaken, is East African cassava mosaic virus (EACMV) (Ndunguru et al., 2005). The virus species are vectored by B. tabaci (Genn.) in a persistent manner, contrasting to CBSIs. Newly-infected plants begin to express symptoms from the top, while plants infected through the planted cutting often show symptoms in all leaves. Symptoms of CMD are a typical mosaic in which there is a mix of yellow/pale green chlorotic patches and green areas (Figure 2D). Unlike CBSD, leaves are usually distorted in shape, and where symptoms are severe the size of leaves is greatly reduced and the plant is stunted. Stunting and the damage to chlorophyll resulting from chlorosis results in the quantitative declines in yield.

BLS is caused by the fungus [Mycosphaerella henningsii (Sivan)]. This fungus is distributed worldwide and typically does not cause great yield loss. The disease manifests in brown circular leaf spots with some varieties expressing a chlorotic halo around the spots. Severe infections can cause the leaves to turn yellow or brown. The circular spots can become dry and crack depending on the environment.

GMD is caused by cassava green mites [Mononychellus tanajoa (Bondar)]. This is a widespread pest in Africa and South America. The mites cause small white, scratch-like spots where they have fed and in severe cases cause the whole leaf to be covered with the pattern. There is such a reduction in chlorophyll that the leaf may become stunted in a manner similar to that caused by CMD. Depending on variety and environment, infestations can lead to losses in tuberous root yield of up to $30 \%$ (Skovgård et al., 1993).

RMD is caused by cassava red spider mite [Oligonychus biharensis (Hirst)], which is widely distributed across Africa. Their feeding damage also causes small scratch-like spotting on the leaf but typically produces a distinct reddish-brown rust color. Feeding is also focused around the main vein but severe infestations can cause the whole leaf to turn orange.

Although GMD and RMD are not strictly diseases, for simplicity, we refer to all of the conditions affecting the plants that were considered in this study as diseases. 


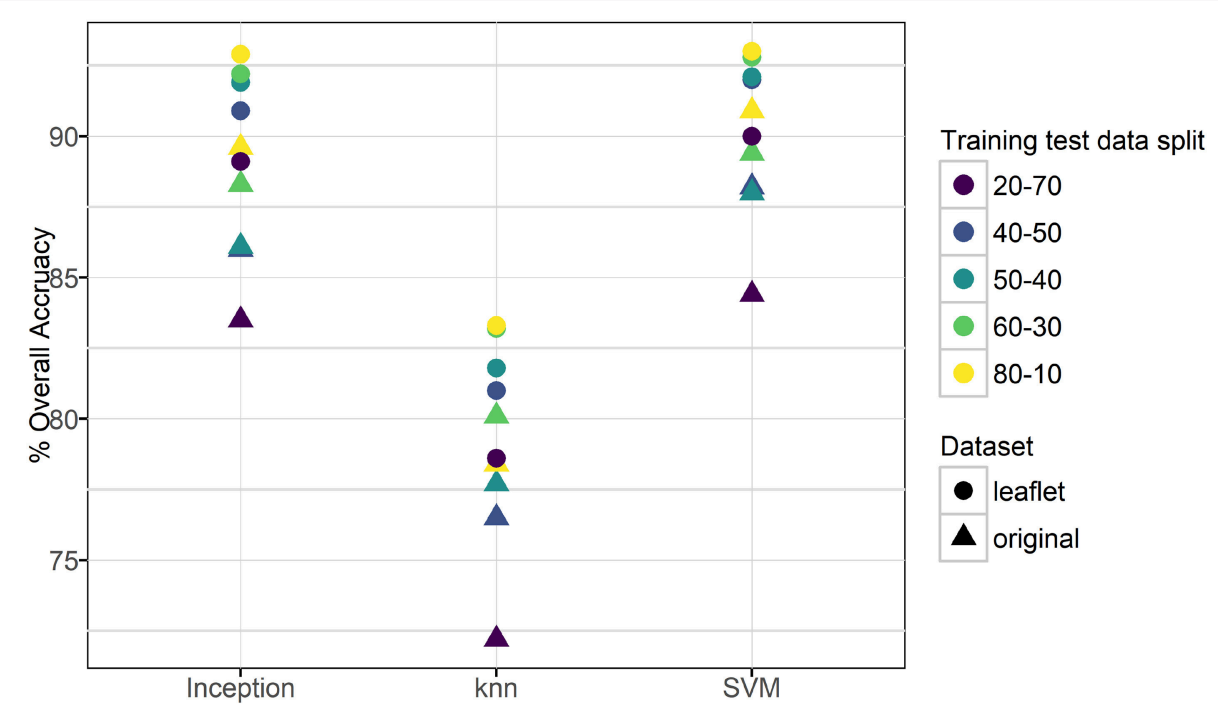

FIGURE 3 | Overall accuracy for transfer learning using three machine learning methods.

\subsection{Approach}

We evaluated the applicability of transfer learning from a deep convolutional neural network (CNN) model for the cassava image datasets. Convolutional neural networks are leading the state-of-the-art in computer vision tasks (Szegedy et al., 2016). As opposed to traditional approaches of training classifiers with hand-designed feature extraction, CNNs learn feature hierarchy from pixels to classifier and train layers jointly. Due to model complexity CNNs can take weeks to fully train, therefore transfer learning is applied to shortcut model training by taking a fully-trained model for a set of classes and retraining the existing weights for new classes. Our approach retrains the existing weights of the Inception v3 CNN model to classify the cassava image datasets by exploiting the large amount of visual knowledge already learned from the Imagenet database. Previous research has shown that transfer learning is effective for diverse applications (Karpathy et al., 2014; Yosinski et al., 2014; Mohanty et al., 2016) and has much lower computational requirements than learning from scratch, which is a benefit to mobile applications.

We analyzed the performance of training the final layer of the CNN model Inception v3 for the new cassava image datasets with three different architectures: the original inception softmax layer, support vector machines (SVM), and knn nearest neighbor $(\mathrm{knn})$. The latest version of the Inception model (based on GoogLeNet), Inception v3, was implemented in TensorFlow. Inception v3 was trained from the ImageNet Large Visual Recognition Challenge using the data from 2012, where it was tasked with classifying images into 1,000 classes. The top- 5 error rate of Inception v3 was $3.46 \%$, compared to 6.67 and $4.9 \%$ for Inception (GoogLeNet) and BN-Inception v2 respectively (Szegedy et al., 2016). Inception v3 is 42 layers deep, but the computation cost is only 2.5 times higher than that of GoogLeNet with 22 layers. Beginning with the GoogLeNet model, Inception v3 implements several design principles to scale up convolutional networks to improve performance with a modest increase in computational cost; a significant benefit for scenarios where memory or computational power is limited, such as mobile or drone devices. Beginning with the GoogLeNet model, Inception v3 factorizes the traditional $7 \times 7$ convolution into three $3 \times$ 3 convolutions, grid reduction is applied to three traditional inception modules to reduce to a $17 \times 17$ grid with 768 filters, then grid reduction is applied again to five factorized inception modules to reduce to a $8 \times 8 \times 1,280$ grid. A detailed description of the design principles implemented to create the Inception v3 model from GoogleNet is provided in Szegedy et al. (2016). The model parameters implemented in this study included the number of training steps $(4,000)$, the learning rate $(0.035)$, train batch size (100), test batch size $(-1$; the entire test set), and the validation batch size (100).

\subsection{Performance Measurements}

In order to perform a robust validation and test for any inherent bias in the datasets, experiments were run for a range of trainingtesting data splits. During model training, $10 \%$ of the dataset was used to validate training steps, thus $90 \%$ of the dataset was split into different training and testing dataset configurations. The training-test splits were as follows: $80-10$ (80\% of dataset for training, $10 \%$ for testing respectively), $60-30$ (60\% of dataset for training, 30\% for testing respectively), 50-40, (50\% of dataset for training, $40 \%$ for testing respectively), $40-50$ (40\% of dataset for training, 50\% for testing respectively), and $20-70$ (20\% of dataset for training, $70 \%$ for testing respectively). For each experiment the overall accuracy is reported as the number of samples in all classes that were similar.

\section{RESULTS}

For the original cassava dataset (i.e., the whole leaf), the overall accuracy in classifying a leaf as belonging to the correct category 


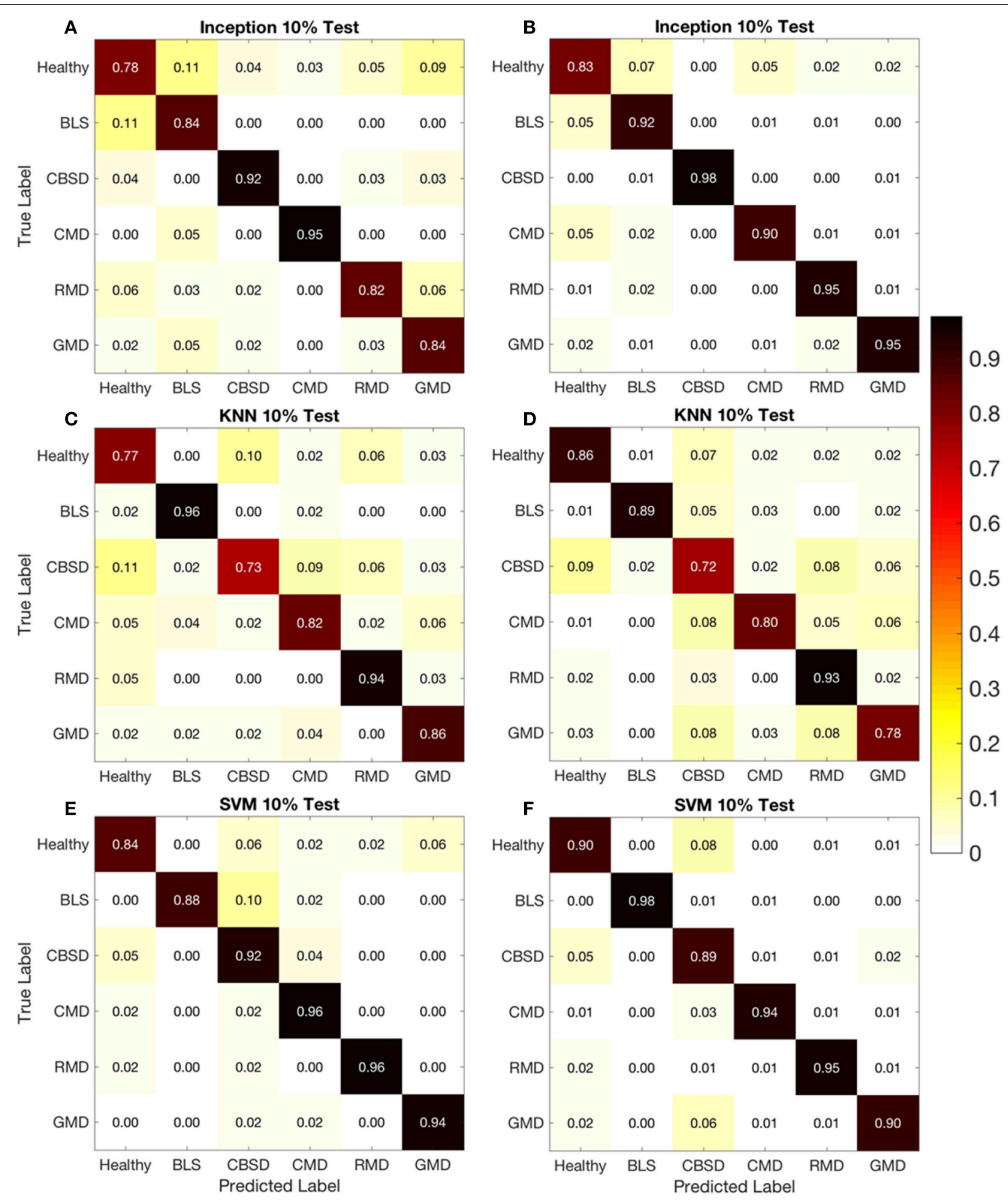

FIGURE 4 | Confusion matrices for 10\% test dataset using original (A,C,E) and leaflet (B,D,F) datasets.

ranged from $73 \%$ (20-70 split, knn) to $91 \%$ (80-10 split, SVM). For the leaflet cassava dataset, the overall accuracy was higher and ranged from $80 \%$ (20-70 split, knn) to $93.0 \%$ (80-10 split, SVM). Figure 3 and Table S2 show the overall accuracies for the datasets. It is worth noting that all models performed much better than randomly guessing, even with varied backgrounds in the images such as human hands, feet, soil or other distracting features. Results also suggest the models were not overfit to the datasets as the training-testing data split had a small effect on the overall accuracies reported. Support vector machines and the final Inception v3 softmax layer, both based on achieving linear separability of the classes, had similar model performances for both original and leaflet datasets, while the knn model $(k=3)$, based on similarity with its neighbors, performed the worst. 
The confusion matrices from the original and leaflet dataset, allow a more detailed analysis by shedding light on how model performance changes with different disease representations in the images. On the confusion matrix plots for the $80-10(80 \%$ of dataset for training, $10 \%$ for testing respectively) data split, the rows correspond to the true class, and the columns show the predicted class. The diagonal cells show the proportion (range $0-1$ ) of the examples the trained network correctly predicts the classes of observations i.e., this is the proportion of the examples in which the true and predicted classes match. The off-diagonal cells show where the network made mistakes.

Using the confusion matrix for the Inception v3 model in Figure 4A (original dataset, 80-10 data split) as an example, the Healthy class diagonal cell shows the Inception v3 model correctly identified 0.78 or $78 \%$ of the healthy leaf images. This increased to 0.83 for the leaflet data set (Figure 4B). Overall the target and output class predictions were within a similar range for all classes and datasets suggesting the overall reported accuracy is indicative of the model performance for all cassava disease classes.

Comparing proportions in the off-diagonal cells for the models showed that the proportion of correct predictions between the leaflet dataset and the original dataset did not significantly increase even though the leaflet dataset was almost 7 times as large as the original dataset. Looking into specific diseases in Figure 4, the highest reported prediction accuracy was 0.98 for CBSD (Inception v3-leaflet) and BLS (SVM- leaflet) diseases. The Inception v3 model had the highest accuracies of 0.98 and 0.95 with the leaflet dataset for CBSD and GMD, respectively. Healthy and BLS classes also had highest accuracies with the leaflet dataset for the SVM model ( 0.90 and 0.98 respectively). The slight improvement in accuracies using the leaflet dataset over the original dataset could be due to the increase in sample size for the disease classes providing more images for the models to learn from. Alternatively, the leaflet dataset could reduce the accuracy of the model as all leaflets on a cassava leaf may not show signs of a disease, which would confuse the model. For CMD and RMD, the SVM model with the original dataset had the highest accuracies of 0.96 and 0.96 , respectively. These results suggest the size of the dataset is not as important in improving prediction accuracy as previously assumed.

\section{DISCUSSION AND CONCLUSION}

The results of this study show that image recognition with transfer learning from the convolutional neural network Inception $\mathrm{v} 3$ is a powerful method for high accuracy automated cassava disease detection. This method avoids the complex and labor-intensive step of feature extraction from images in order to train models, and the model can be easily trained on a desktop and deployed on a mobile device. Transfer learning is also capable of applying common machine learning methods by retraining the vectors produced by the trained model on new class data. In this study, three machine learning methods were used, and results showed the SVM model to be have the highest prediction accuracies for four out of six disease classes. With respect to specific cassava diseases, the SVM model had the highest accuracies for cassava mosaic disease (CMD) (96\%) and red mite damage (RMD) (96\%) using the original dataset, and $90 \%$ and $98 \%$ for healthy and brown leaf spot (BLS) using the leaflet dataset. The Inception v3 model had the highest accuracies for the cassava brown streak disease (CBSD) (98\%) and 95\% accuracy for green mite damage (GMD) with the leaflet dataset.

In a practical field setting where the goal is smartphone assisted disease diagnosis, our results show that diagnostic accuracy improved only slightly when the leaflet was used rather than the whole leaf for some diseases (CBSD, BLS, GMD), while whole leaf images gave higher accuracies for other diseases (CMD and RMD). This was not expected. Rather, the larger image leaflet dataset was expected to perform better for all disease classes compared to the original dataset. These results indicate that datasets needed to build transfer learning models for plant disease diagnosis do not require very large training datasets $(<500$ images per class). The high accuracies reported suggest that variations in background had little effect on the prediction accuracies of the model. Portions of images contained the sky, hands, shoes, and other vegetation, yet predictions in all image classes were greatly above the probability of randomly guessing (16.7\%). In the field it is also likely that an extension worker would use more than one picture to predict the disease, thus improving the diagnostic accuracy further. This study therefore shows that transfer learning applied to the Inception v3 deep learning model offers a promising avenue for in-field disease detection using convolutional neural networks with relatively small image datasets. Work to validate the method in the field with mobile devices has begun through work with TensorFlow Android Inference Interface. Models developed in this study are available on Android devices, shown in the youtube link https://www.youtube.com/watch? $\mathrm{v}=479 \mathrm{p}$-PEubZk\&feature=youtu.be, and are currently being used by researchers in Tanzania to rapidly monitor disease prevalence.

\section{AUTHOR CONTRIBUTIONS}

$\mathrm{AR}, \mathrm{KB}, \mathrm{BA}, \mathrm{JL}$, and $\mathrm{DH}$ conceived the study and wrote the paper. $\mathrm{BA}, \mathrm{KB}$, and JL collected and processed data. PM and AR implemented the algorithms described and prepared results.

\section{FUNDING}

We thank the Huck Institutes at Penn State University for support.

\section{SUPPLEMENTARY MATERIAL}

The Supplementary Material for this article can be found online at: https://www.frontiersin.org/articles/10.3389/fpls.2017. 01852/full\#supplementary-material 


\section{REFERENCES}

Abdullakasim, W., Powbunthorn, K., Unartngam, J., and Takigama, T. (2011). An images analysis technique for recognition of brown leaf spot disease in cassava. Tarım. Makinaları. Bilimi. Dergisi. 7, 165-169.

Aduwo, J. R., Mwebaze, E., and Quinn, J. A. (2010). "Automated vision-based diagnosis of Cassava Mosaic Disease," in Industrial Conference on Data MiningWorkshops (New York, NY), 114-122.

Bellotti, A. C., Smith, L., and Lapointe, S. L. (1999). Recent advances in cassava pest management. Ann. Rev. Entomol. 44, 343-370. doi: 10.1146/annurev.ento.44.1.343

De Bruijn, G., and Fresco, L. (1989). The importance of cassava in world food production. Netherl. J. Agric. Sci. 37, 21-34.

Dubey, S. R., and Jalal, A. S. (2014). Adapted approach for fruit disease identification using images. arXiv preprint arXiv:1405.4930.

FAOSTAT (2017). Faostat:Food and Agriculture Data. Rome: FAOSTAT. (Accessed Jan 04, 2017).

Gibson, D., Burghardt, T., Campbell, N., and Canagarajah, N. (2015). "Towards automating visual in-field monitoring of crop health," in IEEE International Conference on Image Processing (ICIP), 2015 (Quebec City, QC: IEEE), 39063910.

Karpathy, A., Toderici, G., Shetty, S., Leung, T., Sukthankar, R., and Fei-Fei, L. (2014). "Large-scale video classification with convolutional neural networks," in Proceedings of the IEEE conference on Computer Vision and Pattern Recognition (Columbus), 1725-1732.

Legg, J. (1999). Emergence, spread and strategies for controlling the pandemic of cassava mosaic virus disease in east and central africa. Crop Protect. 18, 627-637. doi: 10.1016/S0261-2194(99)00062-9

Legg, J., Owor, B., Sseruwagi, P., and Ndunguru, J. (2006). Cassava mosaic virus disease in east and central africa: epidemiology and management of a regional pandemic. Adv. Virus Res. 67, 355-418. doi: 10.1016/S0065-3527(06)67010-3

Mbanzibwa, D., Tian, Y., Tugume, A., Mukasa, S., Tairo, F., Kyamanywa, S., et al. (2011). Simultaneous virus-specific detection of the two cassava brown streak-associated viruses by rt-pcr reveals wide distribution in east africa, mixed infections, and infections in manihot glaziovii. J. Virol. Methods 171, 394-400. doi: 10.1016/j.jviromet.2010.09.024

Mohanty, S. P., Hughes, D. P., and Salathé, M. (2016). Using deep learning for image-based plant disease detection. Front. Plant Sci. 7:1419. doi: $10.3389 /$ fpls.2016.01419

Mwebaze, E., and Owomugisha, G. (2016). "Machine learning for plant disease incidence and severity measurements from leaf images," in Machine Learning and Applications (ICMLA), 2016 15th IEEE International Conference on (IEEE) (Anaheim, CA).

Nagayet, O. (2005). "Small farms: current status and key trends," in The Future of Small Farms: Proceedings of a Research Workshop (Washington, DC), 355-367.
Ndunguru, J., Legg, J. P., Aveling, T., Thompson, G., and Fauquet, C. M. (2005). Molecular biodiversity of cassava begomoviruses in tanzania: evolution of cassava geminiviruses in africa and evidence for east africa being a center of diversity of cassava geminiviruses. Virol. J. 2:21. doi: 10.1186/1743-422X-2-21

Nichols, R. (1950). The brown streak disease of cassava: distribution, climatic effects and diagnostic symptoms. East Afr. Agric. J. 15, 154-160. doi: $10.1080 / 03670074.1950 .11664727$

Nweke, F. I., Spencer, D. S., and Lynam, J. K. (2002). The Cassava Transformation: Africa's Best-Kept Secret. East Lansing.

Puig, E., Gonzalez, F., Hamilton, G., and Grundy, P. (2015). "Assessment of crop insect damage using unmanned aerial systems: a machine learning approach," in 21st International Congress on Modelling and Simulation (MODSIM2015), (Gold Coast, QLD). Available online at: https://eprints.qut.edu.au/95241/

Siricharoen, P., Scotney, B., Morrow, P., and Parr, G. (2016). "A lightweight mobile system for crop disease diagnosis," in International Conference Image Analysis and Recognition, (Póvoa de Varzim: Springer), 783-791.

Skovgård, H., Tomkiewicz, J., Nachman, G., and Münster-Swendsen, M. (1993). The effect of the cassava green mite mononychellus tanajoa on the growth and yield of cassava manihot esculenta in a seasonally dry area in kenya. Exp. Appl. Acarol. 17, 41-58.

Storey, H. (1936). Virus diseases of east african plants: V.--streak disease of maize. East Afr. Agric. J. 1, 471-475.

Szegedy, C., Vanhoucke, V., Ioffe, S., Shlens, J., and Woina, Z. (2016). "Rethinking the inception architecture for computer vision," in Proceedings of the IEEE Conference on Computer Vision and Pattern Recognition (Las Vegas), 28182826.

Thresh, J., Fargette, D., Otim-Nape, G., et al. (1994). Effects of african cassava mosaic geminivirus on the yield of cassava. Trop. Sci. Lond. 34, 26-26.

Warburg, O. (1894). Die Kulturpflanzen Usambaras. Mitteilungen aus den Deutschen Schutzgebieten 7, 131.

Yosinski, J., Clune, J., Bengio, Y., and Lipson, H. (2014). "How transferable are features in deep neural networks?," in Advances in Neural Information Processing Systems (Montreal), 3320-3328.

Conflict of Interest Statement: The authors declare that the research was conducted in the absence of any commercial or financial relationships that could be construed as a potential conflict of interest.

Copyright (๑) 2017 Ramcharan, Baranowski, McCloskey, Ahmed, Legg and Hughes. This is an open-access article distributed under the terms of the Creative Commons Attribution License (CC BY). The use, distribution or reproduction in other forums is permitted, provided the original author(s) or licensor are credited and that the original publication in this journal is cited, in accordance with accepted academic practice. No use, distribution or reproduction is permitted which does not comply with these terms. 